\title{
Deformation model and experimental evaluation of a contractable and bendable wire-pulling mechanism with embedded soft tubes for a robotic tongue
}

\author{
Nobutsuna Endo*
}

\begin{abstract}
Few physical models of oral and laryngeal systems for human speech movement exist for computer or mechanical simulators. In particular, a robot tongue mechanism that fully reproduces the deformation motion of the human tongue is lacking. The human tongue is an aggregate of muscles that is devoid of a skeleton. It possesses only a small hyoid. A mechanism that can drive and control the deformation of a soft body, such as the human tongue, along multiple degrees of freedom has not been realized to date. To solve this problem, a wire-pulling mechanism with embedded soft tubes is proposed. Using this mechanism, a flexible tongue that can be deformed along multiple degrees of freedom without breaking the wire is achieved. A prototype planar mechanism with two degrees of freedom that is capable of contraction and bending was fabricated. A deformation model that assumes a piecewise constant curvature (PCC) was formulated. Deformation tests confirmed that the prototype is capable of contraction and bending movements that are consistent with those of the model. Variations in the error with respect to the hardness of the deformable part are discussed, and the limits of the deformation model based on the PCC assumption are presented.
\end{abstract}

Keywords: Wire-driven mechanism, Soft mechanism, Tongue, Vocal robot

\section{Introduction}

Motor functions in the human oral and laryngeal systems include speech, mastication, swallowing, and sucking. Analyzing the movements of the oral (pharynx, upper jaw, palate, tongue, lower jaw, teeth, and lips) and laryngeal (around the vocal folds and the epiglottis) systems is more complicated than analyzing the motion of the limbs, trunk, and head. One reason is that most of the organs of the oral system are inside the body; hence, it is impossible to observe these areas visually. Although MRI and X-ray are available, these methods image structures in a stationary state or can measure changes in position and orientation at short intervals. They cannot

\footnotetext{
*Correspondence: noble@ruri.waseda.jp

Tokyo Denki University, 5, Senju Asahi-cho, Adachi-ku, Tokyo 120-8551, Japan
}

measure the forces applied to the organs or movements over extended periods. Some approaches can be reproduced by computer simulation, but because the mechanical properties of each organ are nonlinear, constructing an appropriate comprehensive simulation model has not been successful to date.

An alternative approach for reproducing a physical model of oral and laryngeal systems is to use robots, such as Waseda Talker (WT) [1, 2], vocal [3], or mastication [4] robots. The WT series is limited to reproducing speech functions that are used to utter vowels and some consonants by vibrating the vocal cords and deforming the shape of the vocal tract in the oral cavity. However, the WT model is currently the best reproduction of the human oral and laryngeal systems.

The WT tongue mechanism is based on static MRI measurements of the tongue while individuals uttered 
the five vowels in Japanese. The WT uses a slider-crank drive mechanism and a skin made of a soft material to cover the tongue surface [2]. Therefore, it is not suitable for reproducing tongue shapes and movements with large curvatures, which occur when consonants are uttered. The human tongue is an aggregate of muscles that is devoid of a skeleton but has a small hyoid bone. The human tongue is capable of complex and flexible elongation, contraction, and curvature, during which some tongue muscles contract and others elongate.

Many rod-shaped bending mechanisms, such as those used for catheters, have been proposed for use in continuum robots [5-7]. These robots are equipped with a backbone, allowing them to bend, but they cannot extend, contract, and bend at the same time and cannot perform a tongue movement mechanism. Robotic tongue mechanisms that do not have rigid links inside the tongue include BERO [8], a soft robotic tongue by $\mathrm{Lu}$ et al. [9], ZETS [10], and Anton [11]. In BERO [8], the soft robotic tongue by Lu et al. [9], and ZETS [10], the tongue structure consists of a pneumatic actuator. The intrinsic muscle of the human tongue is an actuator, so these robotic tongue mechanisms more closely simulate the structure of the human tongue. In Anton [11], the actuator is placed outside of the tongue, and the tongue structure consists of a motion converter, which is an advantage for reproducing fast tongue movements. Another advantage is that the Anton mechanism is not limited by the size of the tongue. Anton employs a wire as a flexible motion transmission component that can be placed inside a soft body molded of silicone resin. Simply embedding the wire in the soft silicone resin will cause that the wire tears the resin. In Anton, attaching several beads to the wire prevents it from tearing the resin and converts the wire-pulling motion to a tongue deformation motion. However, Anton's tongue has only one degree of freedom (DOF) because embedding multiple wires with beads during tongue molding is difficult. A wire-pulling mechanism that is used in an anthropomorphic soft hand developed by the author [12] embeds hard tubes in the soft silicone resin to prevent the wire from tearing the resin. Although this mechanism can produce a localized deformation like that of a knuckle, it cannot realize a spatially continuous deformation like that of a tongue.

I have proposed a wire-pulling mechanism with embedded soft tubes (Fig. 1) as a method to incorporate wires into a soft body. This method produces a soft robotic tongue capable of deforming along multiple DOFs without the wire tearing the tongue. In addition, because the soft tube can be fixed and embedded during molding, it is easier to manufacture than an Anton tongue. In a similar study, Lee et al. used a soft tube to pass a shape memory

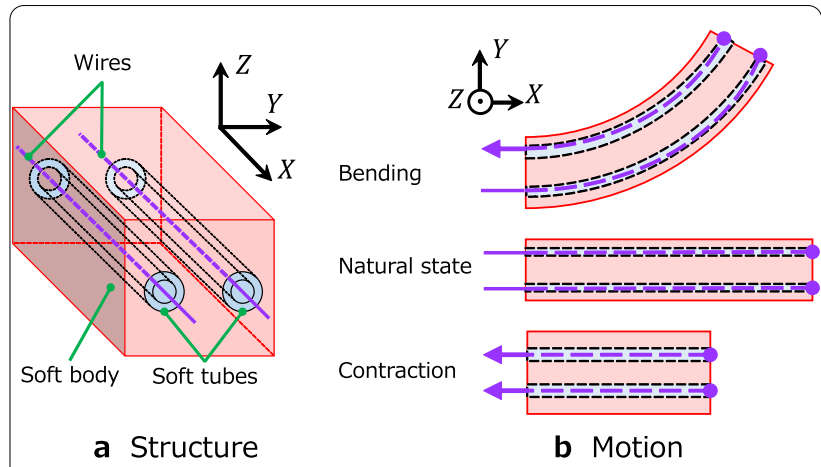

Fig. 1 Schematic diagram of the structure and motion of the planar mechanism with two degrees of freedom. The tension from the combination of two wire enables contraction and bending motions

alloy wire through a soft body [13]. However, the Shore hardness of that soft body was greater than that of A40, which is harder than the soft body used in this study and has better tear resistance. Furthermore, the soft tubes were not used to reduce wear between the wire and the soft body, so the effect of the soft tubes on durability was not assessed.

The purpose of this study is to propose a contraction/ bending wire-pulling mechanism using soft tubes that are embedded in a soft body. A prototype is fabricated to evaluate the deformation produced by the mechanism experimentally. The prototype embodies a planar mechanism with two degrees of freedom. The durability of the prototype and preliminary testing of the deformation produced has been reported [14]. In that study, the error between the modeled and experimental deformation was $\leq 18 \%$. It was not clear whether the difference between the predicted and actual behavior was due to modeling error or inappropriate implementation. In this study, the structure of the mechanism is improved as described in the next section. In addition, deformation tests were conducted using soft body formulations with various hardness values. The sections following are organized as follows: "Wire-pulling Mechanism with Embedded Soft Tubes," "Deformation Modeling," "Deformation Tests," and "Conclusion."

\section{Wire-pulling mechanism with embedded soft tubes}

This section describes the construction of a soft mechanism to realize a robotic tongue. The size of the mechanism to vocalize is constrained by the speed of sound and is a scale-dependent problem because utterance is a phenomenon related to sound waves. A tongue mechanism that mechanically reproduces the natural articulation motion of humans should be of similar size (approximately $70 \times 45 \times 60 \mathrm{~mm}$ ). It is challenging to reproduce 


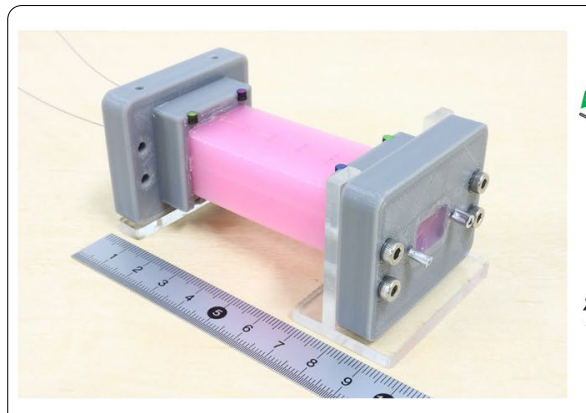

a Appearance

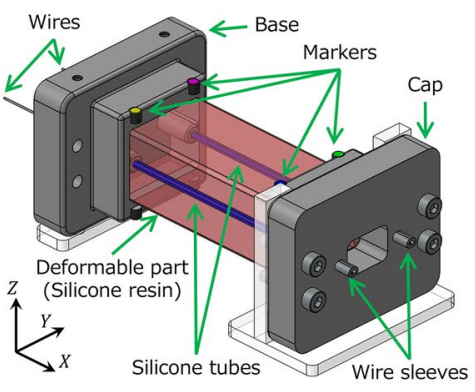

b Structure

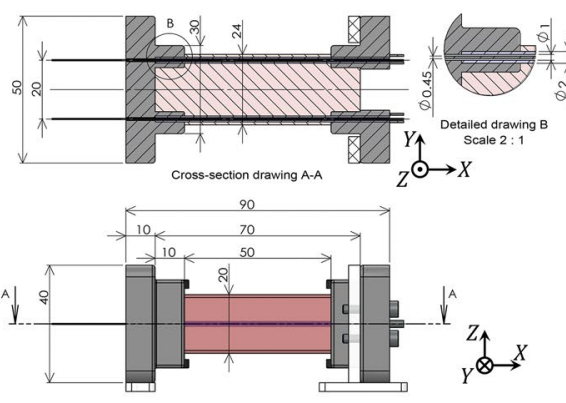

c Main dimension

Fig. 2 Prototype of the planar mechanism with two degrees of freedom

the contraction motion of each intrinsic muscle in the human tongue because the number of such muscles is large, considering the deformation motion that occurs in the sagittal plane is sufficient for motion that is limited to utterance. The WT robot with the best utterance performance at present uses seven degrees of freedom to control the mechanism. This design is based on MRI measurements of the human tongue shape during the utterance of the five Japanese vowels. A design methodology that determines the sufficient number of actuators of a tongue mechanism for utterances of vowels and consonants is yet to be established. This is because it is a complex design problem that involves the range of motion, velocity, and force of the mechanism, and the viscoelasticity of the soft body that occupies the space inside the robot tongue other than the mechanism. In this study, I propose a soft mechanism that can provide up to ten degrees of freedom within a soft body that is the same size as the human tongue.

\section{Structure}

In this paper, I propose a mechanism that embeds soft tubes that are harder than the matrix of the soft body, yet these tubes can elongate, contract, and bend flexibly. Figure 1 illustrates the structure and motion of a planar mechanism with two degrees of freedom that can be contracted and bent. Varying the tensions of the two wires enables contraction and bending. Typically, the soft material that constitutes the central deformable part is damaged by the movement of the wires because it is easily torn. A soft tube that is fabricated with a harder but deformable material is placed between the matrix and the wire to suppress the wear. This soft tube will also experience wear from the movement of the wire, but it is more durable than the matrix.

The prototype shown in Fig. 2 was built to verify the feasibility of this concept. The soft body was manufactured with a silicone resin (Dragon Skin FX-Pro. Smooth-On, Inc.) with a silicone thinner. A silicone tube (Hardness: Shore A60. Outside diameter: $2 \mathrm{~mm}$; inside diameter: $1 \mathrm{~mm}$. MGJG-1×2. Monotaro) was used as the soft tube. The outside diameter of the wire was $0.45 \mathrm{~mm}$ (EA628SS-0.4. ESCO Co., Ltd.). The base and cap were formed with polylactic acid (PLA) resin using a fused deposition modeling (FDM) 3D printer. The wires were pulled by position-controlled motors (RE35. Maxon Motor), harmonic drives (CSF-14-802XH-F. Harmonic Drive Systems Inc.), and pulleys (diameter: $45 \mathrm{~mm}$ ). The maximum tension of the pulley at the stop is $250 \mathrm{~N}$ and the back-drivability and backlash of the harmonic drive are sufficiently small. A previous study [14] employed a Bowden cable mechanism with polytetrafluoroethylene (PTFE) tubes between the base and the driving pulley. However, the backlash in the Bowden cable mechanism could not be ignored, so this mechanism was not used in this study. The Z-axis of the prototype was vertical. A plate was attached to the cap to prevent the cap from tilting in response to the gravitational bending of the deformable part.

The contraction and bending deformations are shown in Fig. 3. A contraction of 50\% of the natural length and a cap angle of $90^{\circ}$ have been achieved. At a plasticizer mixing ratio of $10 \mathrm{wt} \%$, the tensile force required for $90^{\circ}$ bending was about $15 \mathrm{~N}$ when the length of one wire was fixed at the initial length and the other wire was pulled. The deformable part was repeatedly bent by wire tension during durability tests, and the addition of a soft tube increased the number of cycles until the silicone resin was torn by the wire by $>10$ times [14].

\section{Manufacturing}

The manufacturing procedure for the prototype is outlined below and illustrated in Fig. 4.

(1) Insert a stainless-steel rod, having an outer diameter of $1 \mathrm{~mm}$ and a length of $100 \mathrm{~mm}$, into a silicone tube, having a length of $80 \mathrm{~mm}$. Make two of these.

(2) Insert (1) into the holes in the base and cap. 


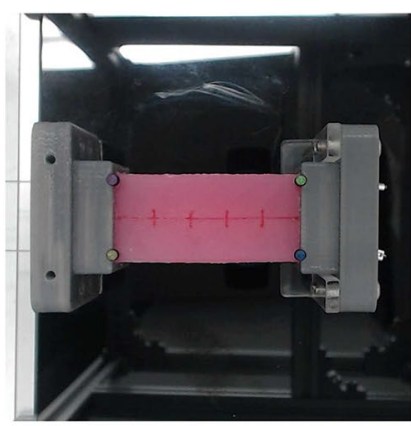

a Natural state

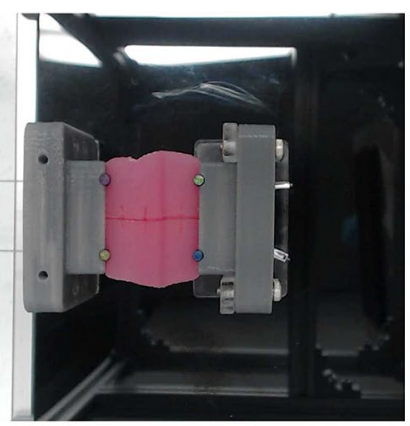

b $50 \%$ contraction

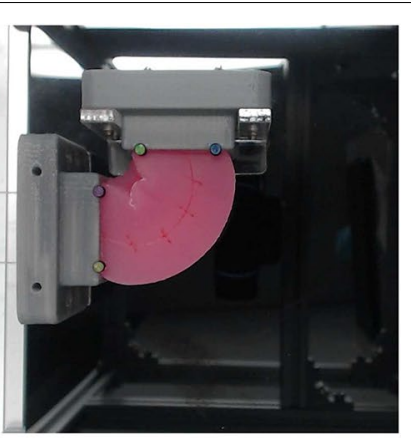

c 90 deg bending

Fig. 3 Contraction and bending deformations of the prototype. The plasticizer mixing ratio is $100 \mathrm{wt} \%$. The wire lengths $I_{1}$ and $I_{2}$ were adjusted manually so that the cap would be at the target position and angle, not by Eq. (3)

(3) Attach molds to (2) and fasten with screws. Block the mold gap with masking tape.

(4) Pour a mixed silicone resin through the gate of the mold. Defoam with a vacuum defoamer if necessary. Wait for the silicone resin to cure.

(5) Remove the sample from the molds when curing is complete. Apply baby powder to decrease the stickiness of the silicone resin surface and wipe the surface with a wet tissue.

(6) Remove the stainless-steel rods.

(7) Enclose each wire with a wire sleeve that is clamp at one end, and pass the wire through each silicone tube.

(8) Attach markers and other accessories.

The wire path in the natural state was straightened by using a stainless steel-rod. It is possible to make the wire path curved in the natural state by using a bent stainless steel rod in step (1) if the wire can be passed through the silicone tube in step (7). Furthermore, a spatial mechanism can be constructed by arranging the wire paths in three dimensions.

\section{Deformation modeling}

A geometric deformation model, such as that of a rigid link mechanism, in which the position and orientation of the mechanism can be calculated from the displacement input to the mechanism, is useful in designing the application of the proposed mechanism to a robotic tongue. In addition, it will be easier to adopt a small and inexpensive servo motor as the drive system, and it can be expected that the control system of a vocal robot will be simplified. A deformation model based on the piecewise constant curvature (PCC) assumption [15] is derived in this section. The deformation model for the $50 \times 24 \times 20 \mathrm{~mm}$ deformable part (Fig. 2) is shown in Fig. 5. Let $d$ be the distance between the wires, and let $L$ be the length of the deformable part when the wire displacements are zero. Let the lengths of the two wires in the deformable part be $l_{1}$ and $l_{2}$, and let the actuator space be $\boldsymbol{q}=\left[\begin{array}{ll}l_{1} & l_{2}\end{array}\right]^{T}$. According to the PCC assumption, the neutral arc line $l$, the wire $l_{1}$, and the wire $l_{2}$ form arcs centered at point $(0, \rho)$. The following equation holds for the radius of curvature $\rho$ using the cap angle $\theta$ :

$$
\left\{\begin{array}{l}
l=\rho \theta \\
l_{1}=\left(\rho-\frac{d}{2}\right) \theta \\
l_{2}=\left(\rho+\frac{d}{2}\right) \theta
\end{array}\right.
$$

Solving this yields a transformation from the actuator space to the configuration space $\boldsymbol{c}=\left[\begin{array}{ll}l & \theta\end{array}\right]^{T}$.

$$
\boldsymbol{c}(\boldsymbol{q})=\left[\begin{array}{l}
l(\boldsymbol{q}) \\
\theta(\boldsymbol{q})
\end{array}\right]=\left[\begin{array}{cc}
\frac{1}{2} & \frac{1}{2} \\
-\frac{1}{d} & \frac{1}{d}
\end{array}\right]\left[\begin{array}{l}
l_{1} \\
l_{2}
\end{array}\right]
$$

Likewise, the actuator space can be transformed from the configuration space by the following equation:

$$
\boldsymbol{q}(\boldsymbol{c})=\left[\begin{array}{l}
l_{1}(\boldsymbol{c}) \\
l_{2}(\boldsymbol{c})
\end{array}\right]=\left[\begin{array}{cc}
1 & -\frac{d}{2} \\
1 & \frac{d}{2}
\end{array}\right]\left[\begin{array}{l}
l \\
\theta
\end{array}\right]
$$

The transformation from the configuration space to the task space $\boldsymbol{p}=\left[\begin{array}{ll}x & y\end{array}\right]^{T}$ is achieved as follows:

$$
\left\{\begin{array}{l}
x=\rho \sin \theta=\frac{l}{\theta} \sin \theta \\
y=\rho(1-\cos \theta)=\frac{l}{\theta}(1-\cos \theta)
\end{array}\right.
$$

Because $\sin \theta \approx \theta, \cos \theta \approx 1-\frac{\theta^{2}}{2}$ when $\theta$ is very small, $\boldsymbol{p}=\left[\begin{array}{ll}x & y\end{array}\right]^{T}$ is represented by the following approximation.

$$
\left\{\begin{array}{l}
x=\frac{l}{\theta} \sin \theta \approx \frac{l}{\theta} \theta=l \\
y=\frac{l}{\theta}(1-\cos \theta) \approx \frac{l}{\theta}\left\{1-\left(1-\frac{\theta^{2}}{2}\right)\right\}=\frac{l \theta}{2}
\end{array}\right.
$$




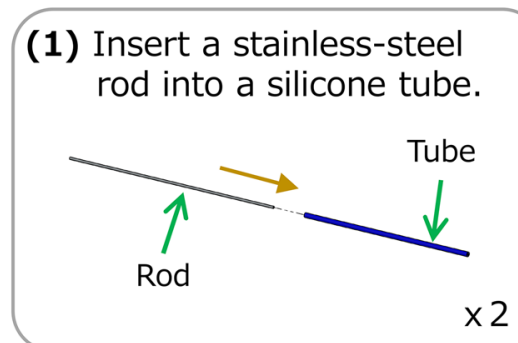

(3) Attach molds.

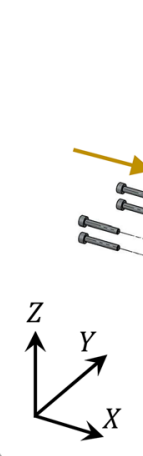

(6) Remove the rods.

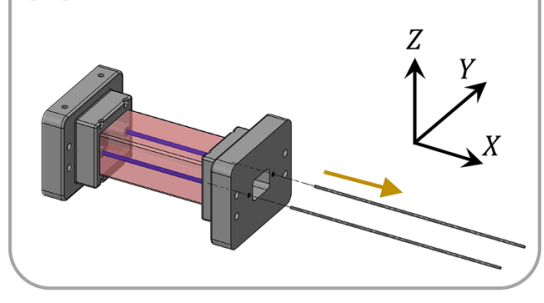

(2) Insert (1) into the holes in the base and the cap.

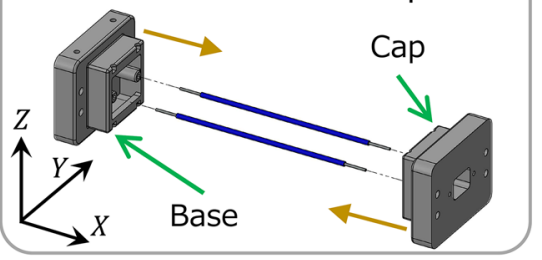

(4) Pour a mixed silicone resin.

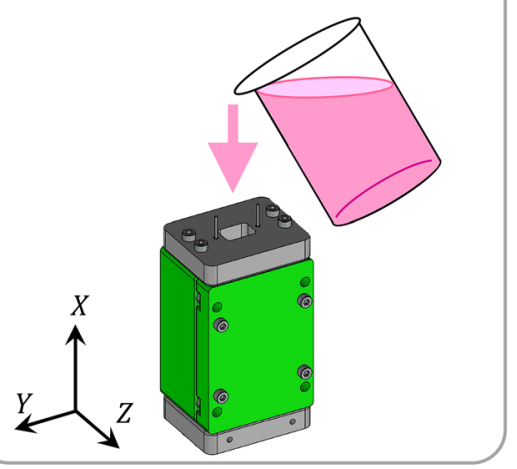

(7) Pass wires through the tubes.

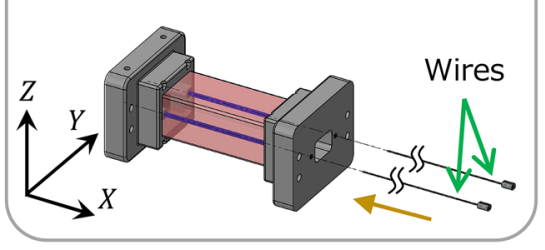

(5) Remove from the molds.

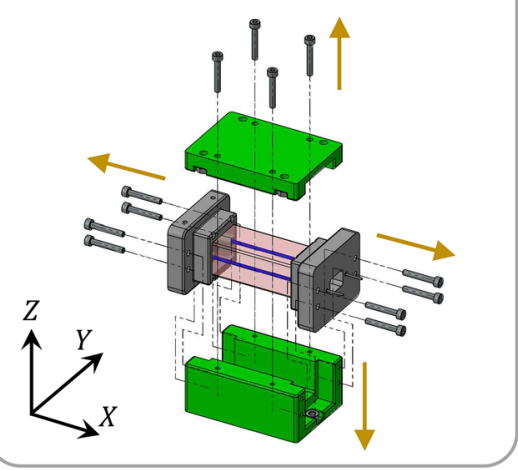

(8) Attach markers etc.

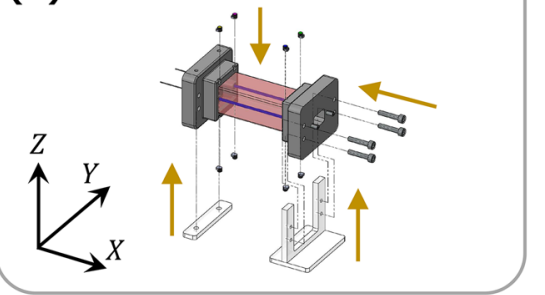

Fig. 4 Manufacturing procedure

\section{Deformation tests}

I tested whether the prototype was deformed according to the model based on the PCC assumption. As noted in the "Wire-pulling Mechanism with Embedded Soft Tubes" section, a design method for determining the hardness of a soft body suitable for a robotic tongue, independent of the mechanical structure, has not been established. Therefore, I tested whether the prototype deforms according to the model when the hardness of the soft body is varied. The hardness of the silicone resin (Dragon Skin FX-Pro. Smooth-On, Inc.) can be adjusted by varying the amount of plasticizer (Silicone Thinner. Smooth- On, Inc.). The total weight $W$ of the formulated silicone resin is the sum of the weights of the base resin $W_{A}(\mathrm{~g})$, the curing agent $W_{B}(\mathrm{~g})$, and the plasticizer $W_{T}$ (g): $W=W_{A}+W_{B}+W_{T}$ (g). The mixing ratio of the plasticizer $r_{m}$ (wt\%) is defined by the following equation:

$$
r_{m}=\frac{W_{T}}{W_{A}+W_{B}} \times 100
$$

In the deformation tests described below, experiments were conducted with plasticizer mixing ratios of 10,25 , 50,75 , and $100 \mathrm{wt} \%$. The durometer hardness and elastivity/compliance of the silicone resin at these plasticizer mixing ratios have been previously reported $[16,17]$.

\section{Experimental setup}

The experimental setup is shown in Fig. 6. The two wires of the prototype are pulled by the position-controlled motor and pulley. The deformation of the prototype was captured by a USB camera (C922 Pro Stream Webcam. Logicool). The camera was calibrated in advance, and the captured image was corrected for distortion. The cap position $\boldsymbol{p}=\left[\begin{array}{ll}x & y\end{array}\right]^{T}$ and cap angle $\theta$ relative to the base 
were calculated from the measured marker positions of the base and cap. The measurement resolution of the marker position was $0.135 \mathrm{~mm} / \mathrm{px}$. The neutral arc length $l$ of the deformable part when PCC is assumed was calculated by the following equation, which was derived from Eqs. (4) and (5):

$$
l= \begin{cases}x & \left(\theta<5^{\circ}\right) \\ \frac{x \cdot \theta}{\sin \theta} & \text { otherwise })\end{cases}
$$

Two types of deformation patterns were tested: (a) pulling only $l_{1}$ and (b) pulling both $l_{1}$ and $l_{2}$. In deformation pattern (a), the length of one wire, $l_{2}=L=50 \mathrm{~mm}$, is constant and the input is $l_{1}$ such that the reference value of the cap angle $\theta_{r}$ is $0^{\circ}, 30^{\circ}, 60^{\circ}$, and $90^{\circ}$. In deformation pattern (b), the inputs are $l_{1}$ and $l_{2}$ such that the reference values of the neutral arc length and the cap angle $\left(l_{r}, \theta_{r}\right)$ are $50 \mathrm{~mm}, 0^{\circ} ; 40 \mathrm{~mm}, 15^{\circ} ; 40 \mathrm{~mm}, 30^{\circ} ; 30 \mathrm{~mm}, 30^{\circ}$; and $30 \mathrm{~mm}, 60^{\circ}$. The wires were moved at constant speeds to the set values of the wire lengths, stopped for $1 \mathrm{~s}$, and then moved to the next set values. Images were taken at $0.5 \mathrm{~s}$ after stopping at each setpoint. For each deformation pattern, pulling and return measurements were obtained three times.

\section{Results}

Images of deformation pattern (a), where $l_{1}$ is pulled, are shown in Fig. 7, and the graphs of the trajectories in configuration space $\boldsymbol{c}=\left[\begin{array}{ll}l & \theta\end{array}\right]^{T}$ and task space $\boldsymbol{p}=\left[\begin{array}{ll}x & y\end{array}\right]^{T}$, the neutral arc length $l$, and cap angle $\theta$ are shown in Figs. 8, 9, 10. A linear trajectory in the configuration space and an arc-shaped trajectory in the task space are drawn in Fig. 8; these are similar to the deformation model. The neutral arc length $l$ decreases proportionally with decreasing wire length $l_{1}$, as shown in Fig. 9, which is also similar to the deformation model. The error and relative error of the neutral arc length $l$ tend to increase with decreasing wire length $l_{1}$ when the plasticizer mixing ratio is between 10 and $50 \mathrm{wt} \%$. The cap angle $\theta$ also increases proportionally as wire length $l_{1}$ decreases (Fig. 10), as in the deformation model. The error and relative error of the cap angle $\theta$ tend to increase as wire length $l_{1}$ decreases.

The root mean square (RMS) of the errors and the relative errors are shown in Fig. 11 to evaluate the effect of the hardness of the deformable part. The error of the neutral arc length $l$ during pulling decreases as the plasticizer mixing ratio increases (Fig. 11a). However, the differences between the errors during pulling and return increase as the plasticizer mixing ratio increases. Consequently, the error in return decreases until the plasticizer

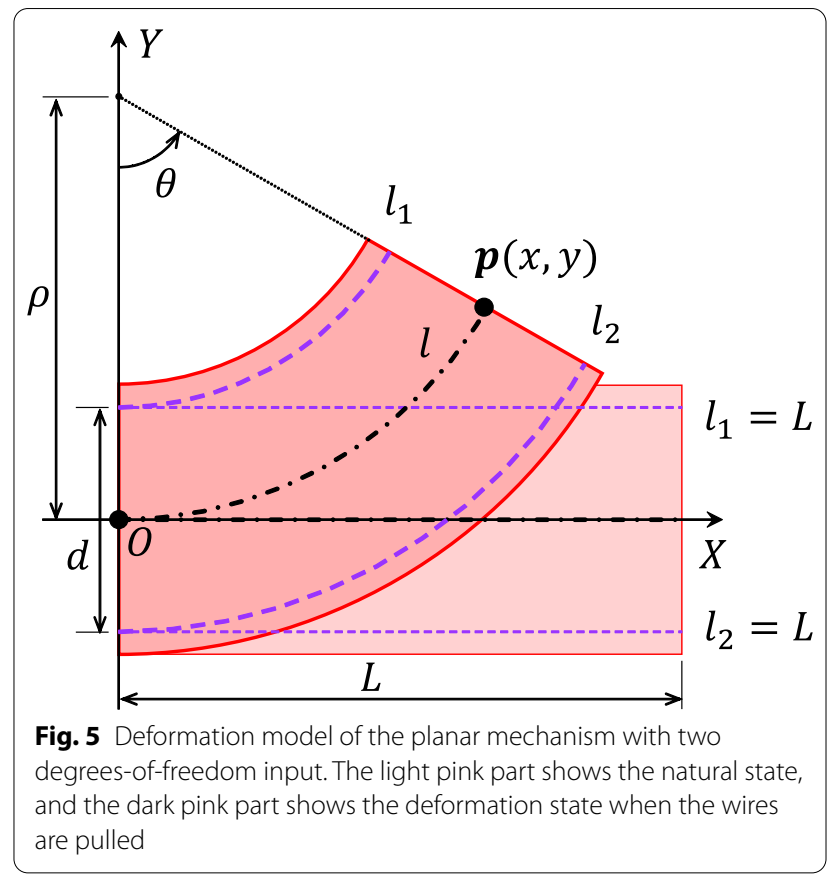

mixing ratio is $75 \mathrm{wt} \%$, but increases at $100 \mathrm{wt} \%$. The error of the cap angle $\theta$ was large only when the plasticizer mixing ratio was $10 \mathrm{wt} \%$; at other ratios, the error was almost constant (Fig. 11b). In addition, there was no difference between the errors during pulling and return.

Images of deformation pattern (b), where both $l_{1}$ and $l_{2}$ are pulled, and the graphs of the trajectories in the configuration space $\boldsymbol{c}=\left[\begin{array}{ll}l & \theta\end{array}\right]^{T}$ and task space $\boldsymbol{p}=\left[\begin{array}{ll}x & y\end{array}\right]^{T}$, the neutral arc length $l$, and cap angle $\theta$ are shown in Figs. $12,13,14,15$. In the configuration space, $l$ and $\theta$ initially decrease, then $\theta$ increases while $l$ remains constant, then $l$ decreases while $\theta$ remains constant, and finally $\theta$ increases while $l$ remains constant (Fig. 13). This pattern is in agreement with the deformation model. The neutral arc length $l$ and cap angle $\theta$ decrease and increase, respectively, as the wire length $l_{1}$ decreases (Figs. 14 and 15), as in the deformation model. The error and relative error of the neutral arc length $l$ exhibit an approximately linear increase as wire length $l_{1}$ decreases (Fig. 14). The error and relative error of the cap angle $\theta$ tend to increase as wire length $l_{1}$ decreases (Fig. 15).

The RMS values for the error and the relative error that show the effect of the hardness of the deformable part are shown in Fig. 16. The trend is the same as that of deformation pattern (a). As the plasticizer mixing ratio increases, the error of the neutral arc length $l$ decreases; however, the difference in the values of $l$ between pulling and return increases. In contrast, there 


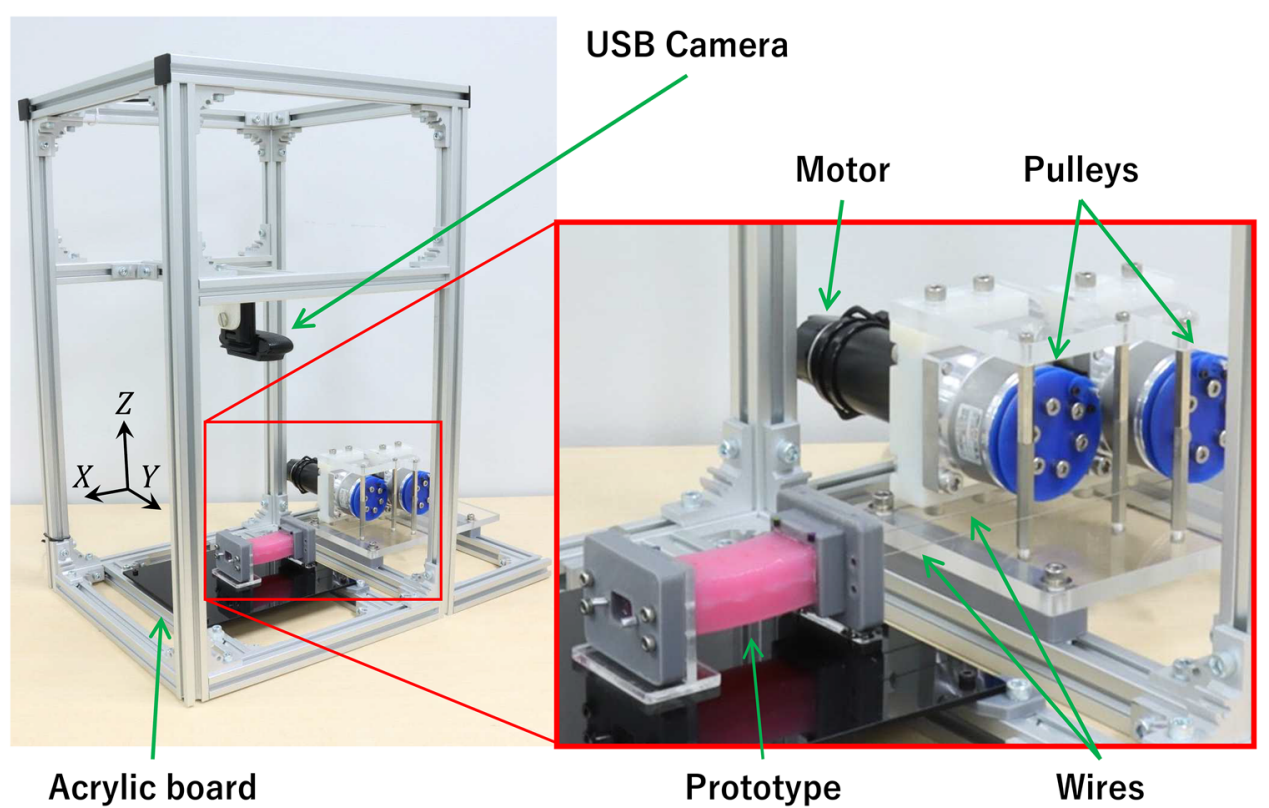

Fig. 6 Experimental equipment. The base is fixed to a black acrylic plate which is fixed to the frame. The cap can be moved planarly on the black acrylic plate

is no difference between the cap angle $\theta$ values during pulling and return.

\section{Discussion}

The prototype was deformed in the same way as the model, and the effectiveness of representing the mechanism of the experimental deformation with an arc-shaped deformation model based on the PCC assumption was demonstrated. In a previous report [14], the change in the cap angle with respect to the wire length $l_{1}$ for deformation pattern (a) was reported for a prototype with a plasticizer mixing ratio of 50 $\mathrm{wt} \%$. The maximum relative error was $-17.4 \%$ when $\theta_{r}=90^{\circ}$, and the RMS of the relative error during pulling was $14.6 \%$. Using the same conditions, the relative error in this study is $-13.7 \%$, and the RMS of the relative error during pulling is $10.4 \%$. As mentioned in the "Wire-pulling Mechanism with Embedded Soft" section, the previous study used a Bowden cable mechanism between the driving pulley and the base. The decision to omit the Bowden cable mechanism appears to have contributed to the reduction in error. In the graphs of trajectories in the configuration space and task space and errors, the mean values of three trials were plotted and the standard deviations were drawn as an error bar. The standard deviation is sufficiently

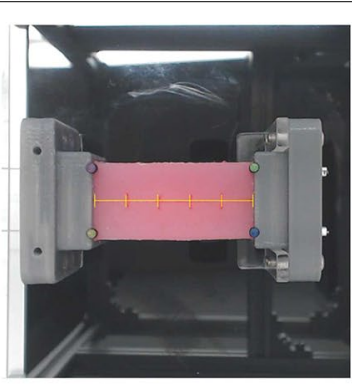

a $\theta_{r}=0^{\circ}$

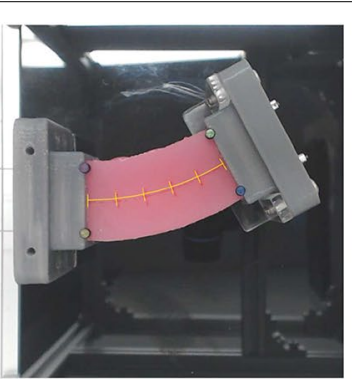

b $\theta_{r}=30^{\circ}$

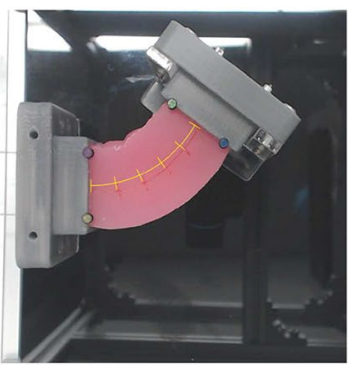

c $\theta_{r}=60^{\circ}$

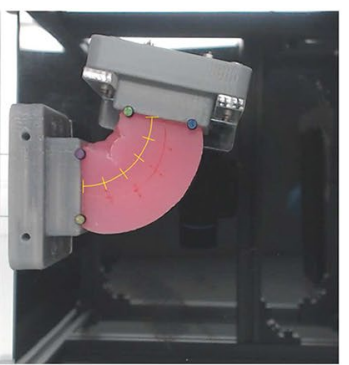

d $\theta_{r}=90^{\circ}$

Fig. 7 Pictures of deformation pattern (a) when only $I_{1}$ is pulled. The plasticizer is used at 100 wt $\%$. The yellow line indicates the neutral arc line / on the surface of the deformable part predicted by the deformation model. Note that the red line on the surface of the prototype does not match the neutral arc line in the plane where the wires exist because the prototype does not deform uniformly in the Z-direction 

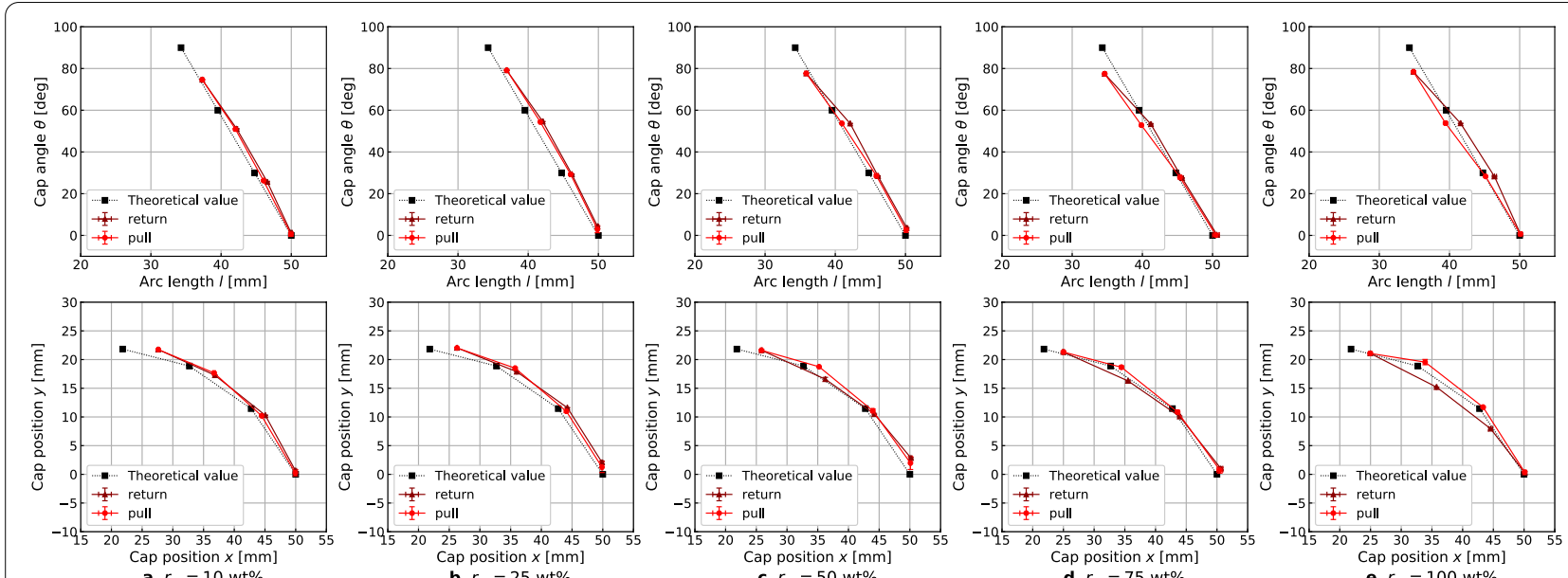

Fig. 8 Trajectories in configuration space $c=\left[\begin{array}{ll}l & \theta\end{array}\right]^{T}$ (top) and task space $p=\left[\begin{array}{ll}x & y\end{array}\right]^{T}$ (bottom) of deformation pattern (a). Each plot represents the mean value of the three times. The error bars represent the standard deviation.

small compared to the magnitude of the error that the the neutral arc length $l$, the RMS values of the error
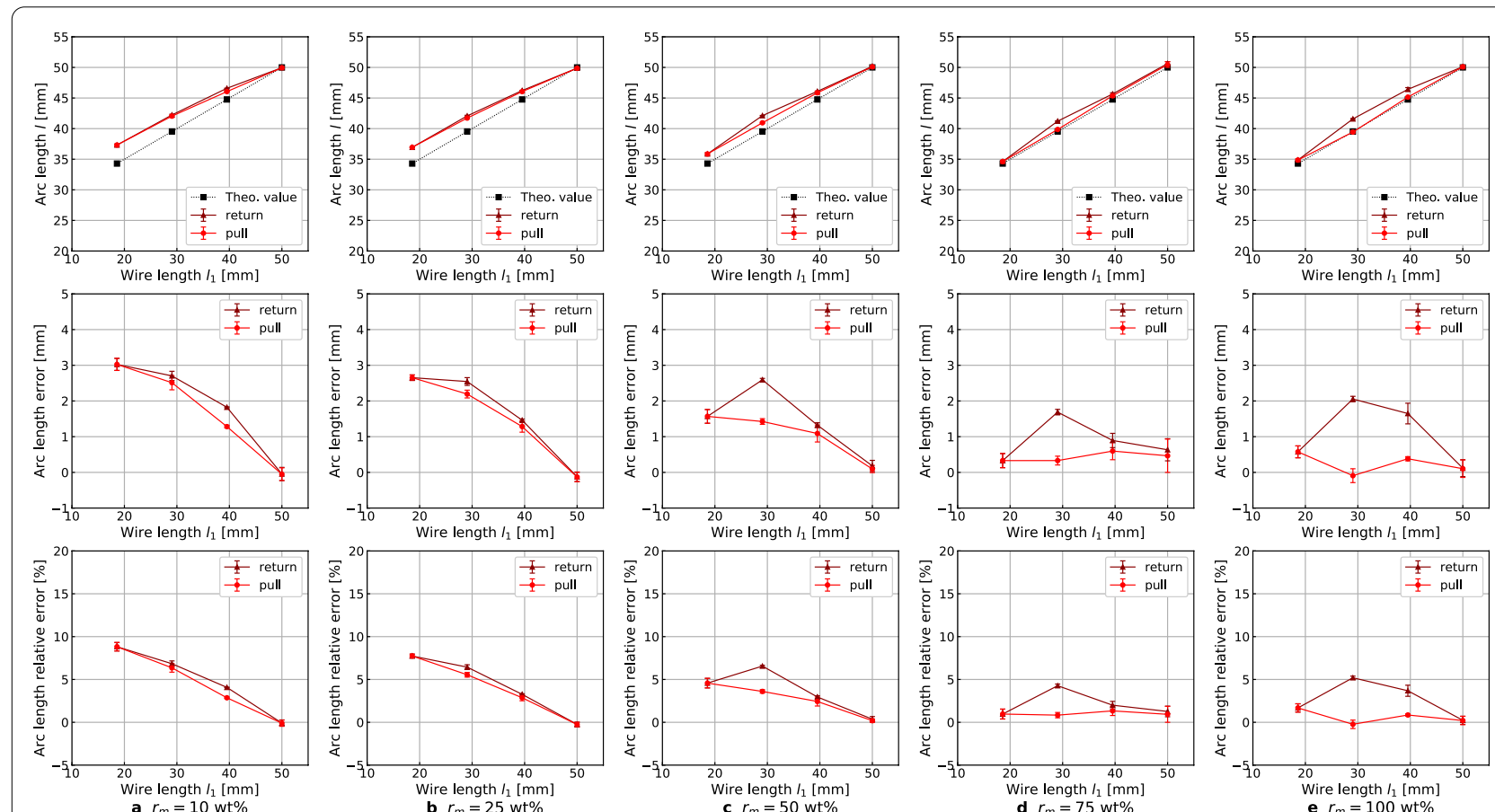

Fig. 9 Neutral arc length / (top), its error (middle), and relative error (bottom) to wire length /, for each plasticizer mixing ratio in deformation pattern (a). Each plot represents the mean value of the three times. The error bars represent the standard deviation

number of repetitions of pulling and returning does not seem to have a significant effect on the error.

However, the relative error remains approximately $10 \%$, indicating that the arc-shaped deformation model based on the PCC assumption is limited. For decrease as the deformable part becomes softer during pulling in either of the two deformation patterns tested (Figs. 11 and 16). In particular, the RMS with a plasticizer mixing ratio of $100 \mathrm{wt} \%$ in deformation pattern (a) is almost equal to the measurement error observed 

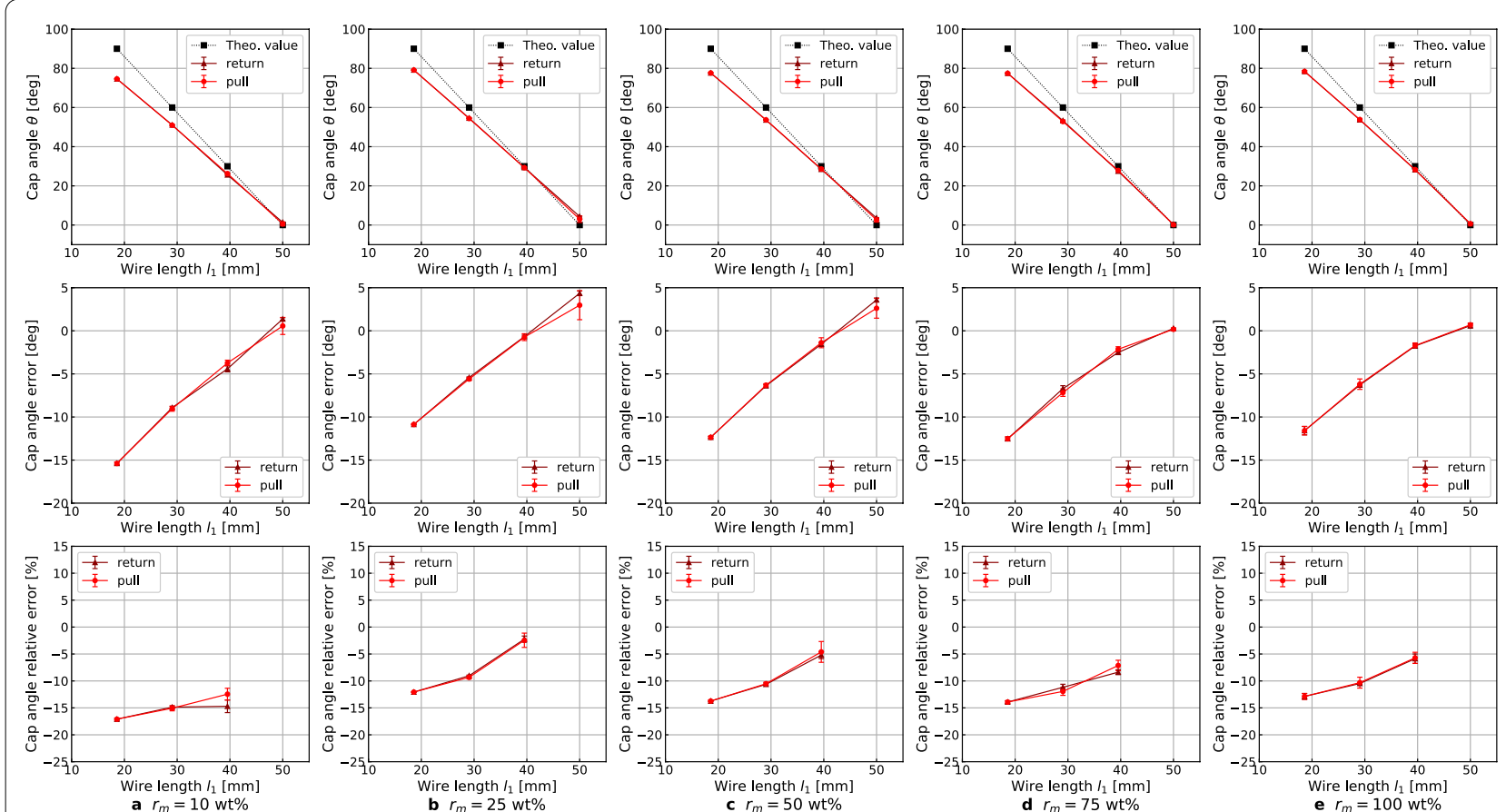

Fig. 10 Cap angle $\theta$ (top), its error (middle), and relative error (bottom) to wire length //for each plasticizer mixing ratio in deformation pattern (a). Each plot represents the mean value of the three times. The error bars represent the standard deviation

in the image, which is consistent with the deformation model. Therefore, adding a correction term for the hardness of the deformable part to the arc-shaped deformation model under the PCC assumption could counteract this limitation. In contrast, for the cap angle $\theta$, the RMS values of the error and relative error are constant when the plasticizer mixing ratio is increased in both deformation patterns. However, the plasticizer ratio of $10 \mathrm{wt} \%$ is an exception. Because a wire is used in this mechanism, it is possible that the wire is not tangentially connected before and after the end face of the deformable part; rather, it flexes. In addition, although the radial deformation of the circular arc is ignored in the deformation model, a radial strain due to the Poisson effect occurs in the matrix of the deformable part. Such a strain would lengthen the wire path relative to that predicted for the arc-shaped path by the deformation model and may be a cause of the error that exists independently of hardness.

Hysteresis (i.e., the difference between pulling and return) increased as the plasticizer mixing ratio increased. The wire exerts a force only during compression of the deformable part; the wire does not contribute to the extension of the deformable part. The elastic force of the deformable part contributes to the return. Therefore, if the plasticizer mixing ratio is large, and the elasticity of the deformable part is low,
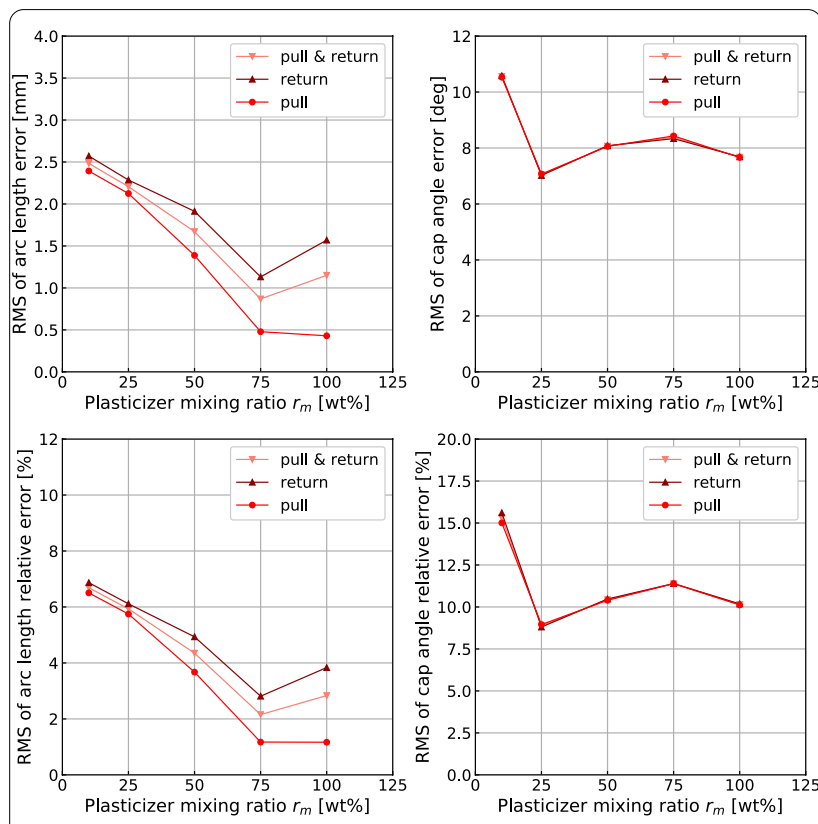

a Arc length $I$

b Cap angle $\theta$

Fig. 11 Root mean square of error to plasticizer mixing ratio in deformation pattern (a) 

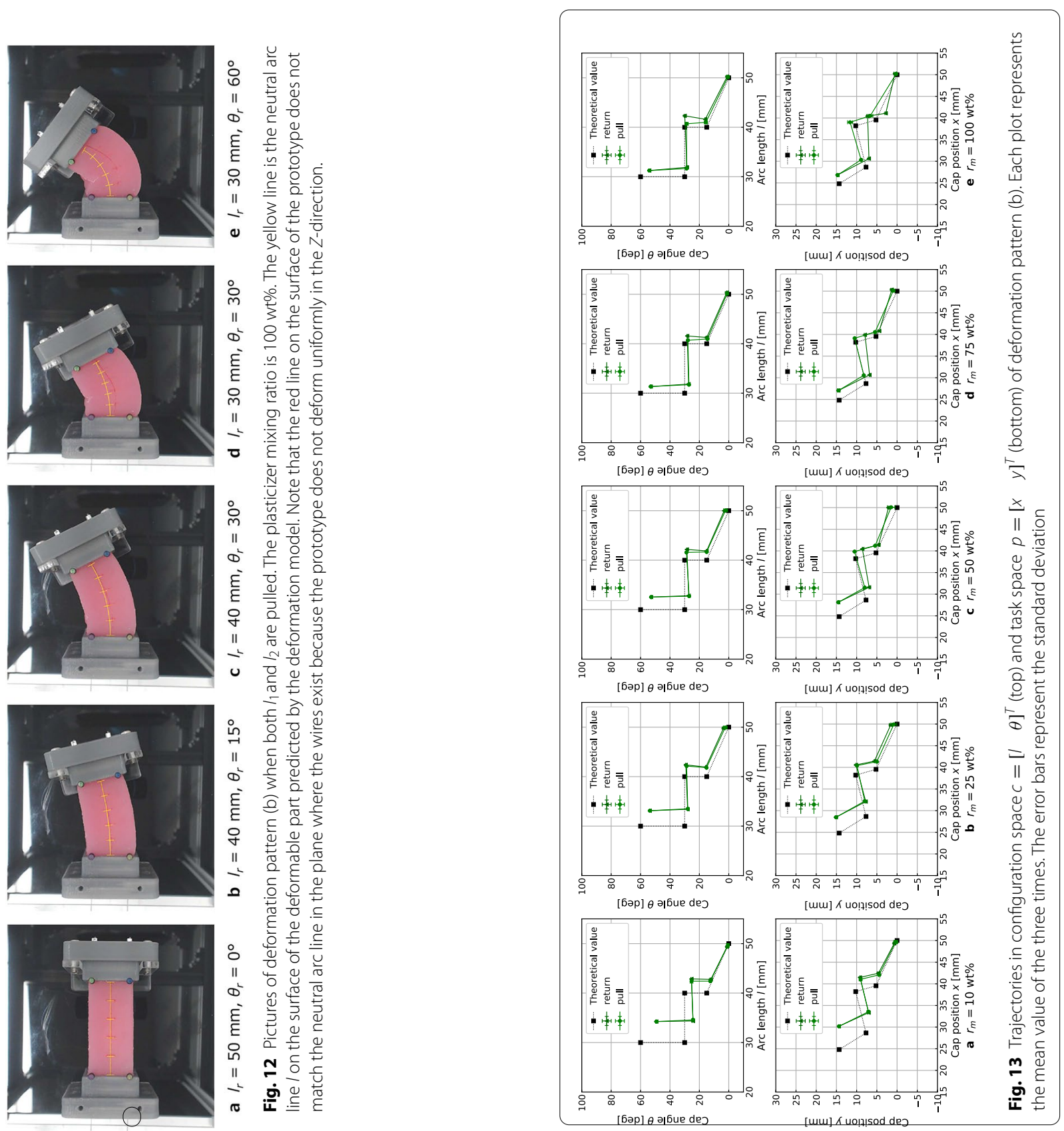

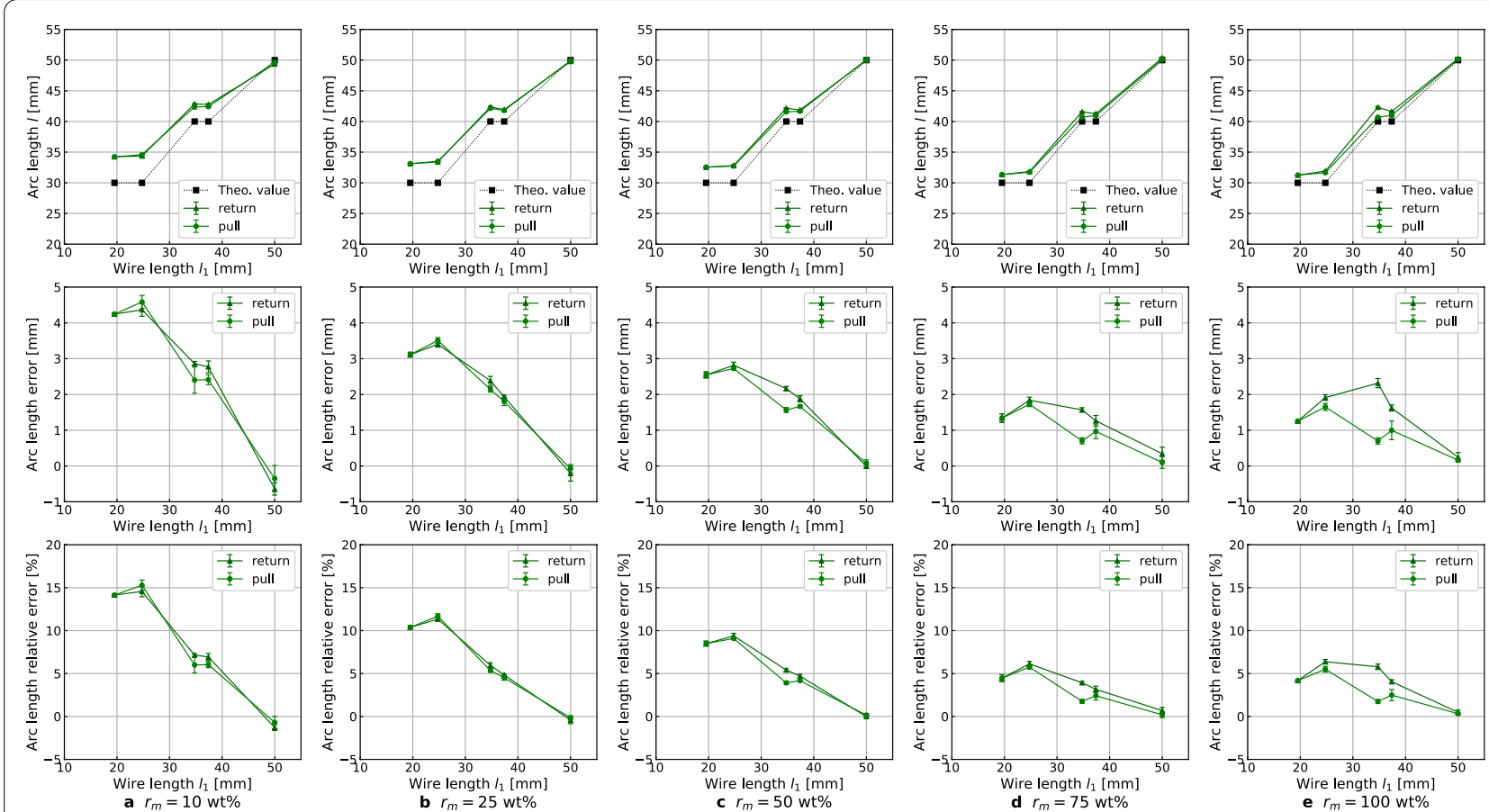

Fig. 14 Neutral arc length / (top), its error (middle), and relative error (bottom) to wire length / for each plasticizer mixing ratio in deformation pattern (b). Each plot represents the mean value of the three times. The error bars represent the standard deviation
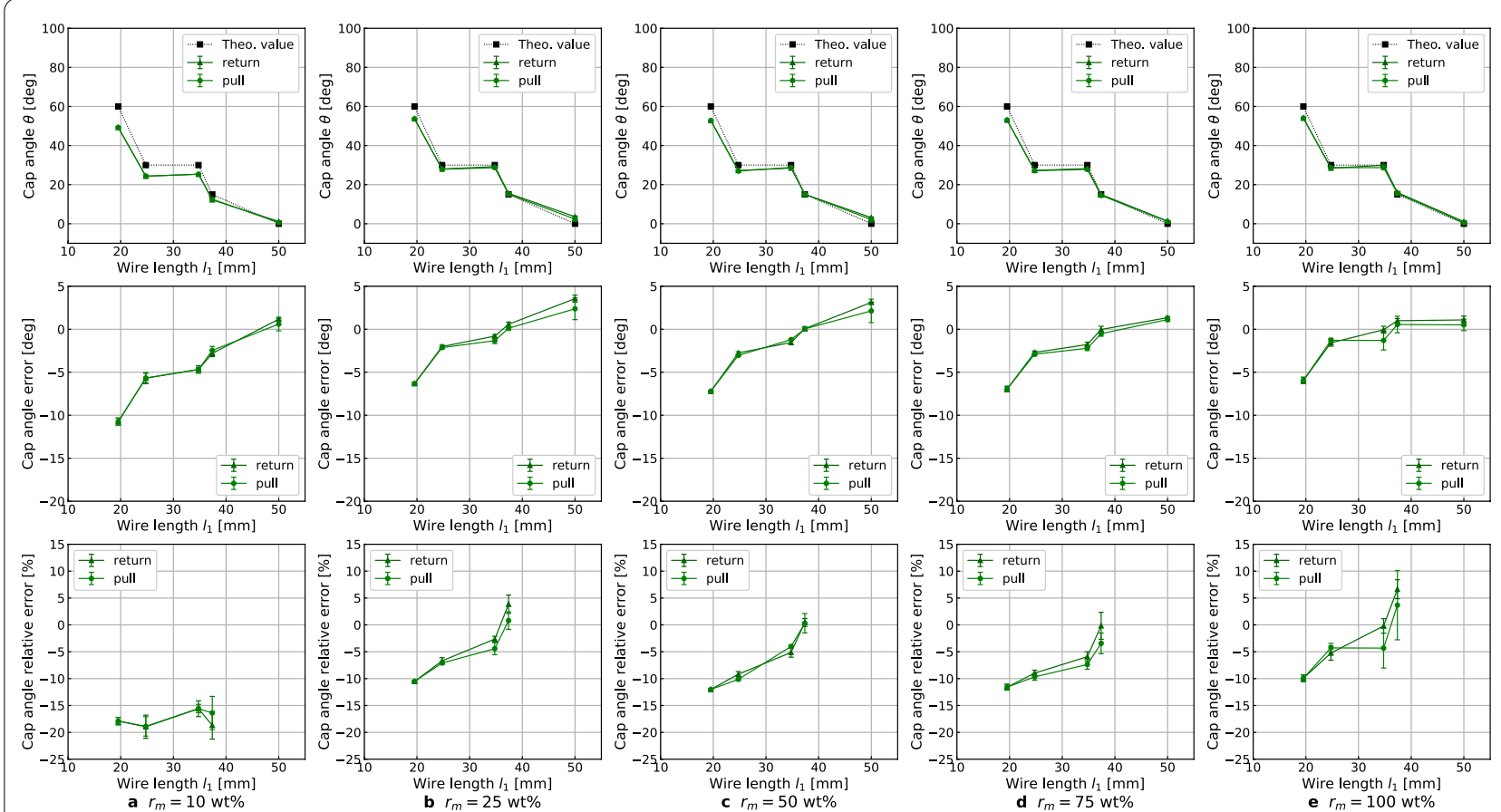

Fig. 15 Cap angle $\theta$ (top), its error (middle), and relative error (bottom) to wire length $/ 1$ for each plasticizer mixing ratio in deformation pattern (b). Each plot represents the mean value of the three times. The error bars represent the standard deviation 

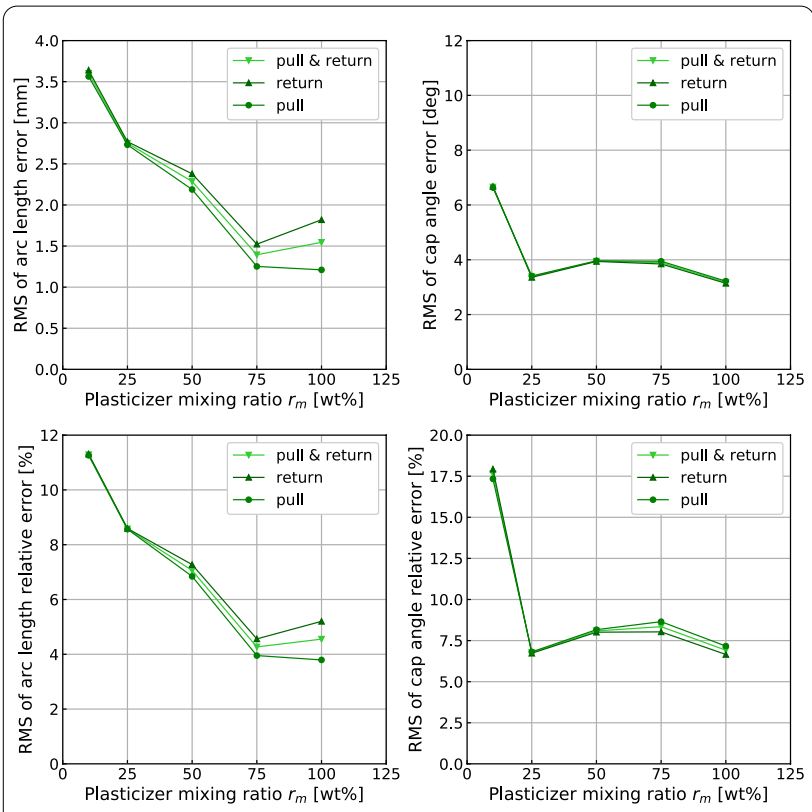

a Arc length $I$

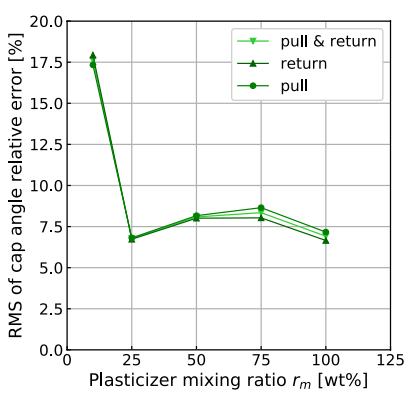

b Cap angle $\theta$

Fig. 16 Root mean square of error to plasticizer mixing ratio in deformation pattern (b)

then hysteresis may be caused by an relative increase in the frictional force between the cap plate and the black acrylic plate. When this mechanism is applied to a vocal robot tongue, the planar constraint of this prototype is not necessary, and the $\mathrm{Y}$ direction shown in Fig. 5 is the vertical direction. Consequently, the effect of hysteresis is considered to be negligible. However, when the plasticizer mixing ratio is large, the deformation caused by the weight of the deformable part must be considered.

In the proposed mechanism, position-controlled motors with sufficient servo stiffness pull the wires (steady-state deviation of the motor angle was less than $0.5 \%$ ); therefore, the input is a displacement, not a force. For this reason, the deformation is modeled by geometry without solving for the static force equilibrium in this study. Including the hardness of the deformable part as a parameter would increase the accuracy of the deformation model. In this case, the equilibrium between the displacement due to the wire and the elastic force of the deformable part must be considered. In [18], modeling was performed by balancing the pulling force and the elastic force of the cantilever beam, while assuming PCC. However, in the mechanism presented in this paper, the wire to be pulled is not a flexible beam. Instead, the matrix of the deformable part exerts an elastic force, making it difficult to apply the method of [18]. In [5, 6], elastic beams are bent by wires, and deformation models that consider friction are established without assuming PCC. In contrast, in this study, a sufficiently thick elastic mass is used instead of an elongated flexible beam that does not contract; therefore, this application is still difficult. More rigorous deformation modeling is a future challenge.

In this study, the deformation was measured with a camera. However, when applied to the tongue mechanism of a vocal robot, it is difficult to measure deformation using a camera. Extension/contraction/bending sensors or IMUs that can be incorporated into a soft body made of silicone resin is also an improvement that is needed in the future.

\section{Conclusion}

The aim of this work was to develop a tongue mechanism that can be mounted on a vocal robot. In this paper, I proposed a wire-pulling mechanism with embedded soft tubes as a method to incorporate wires into soft material. A prototype capable of contraction and bending was built, and the manufacturing method was described. A deformation model based on the PCC assumption was formulated. The prototype was evaluated in the same way as the model in deformation tests, demonstrating the effectiveness of the model. Variations in the error with respect to the hardness of the deformable part were discussed, and the limits of a deformation model based on the PCC assumption were presented.

More accurate deformation modeling that includes forces and viscoelasticity characteristics of the mechanism and extension/contraction/bending sensors that can be incorporated into the soft body will be investigated in future studies. A mechanism with two degrees of freedom is not suitable for use as a tongue mechanism of a vocal robot. The construction of deformation models and control systems with more degrees of freedom will be pursued in future studies.

\section{Acknowledgements}

Not applicable.

\section{Authors' contributions}

NE proposed the wire-pulling mechanism with embedded soft tubes, built the prototype, formulated the deformation model, conducted the experiments, analyzed the results, wrote the manuscript. The author read and approved the final manuscript.

\section{Funding}

This work was partially supported by JSPS KAKENHI Grant-inAid for EarlyCareer Scientists $18 \mathrm{~K} 13717$ and Research Institute for Science and Technology of Tokyo Denki University Grant Number QT17T-04.

\section{Availability of data and materials}

The datasets used and analysed during the current study are available from the corresponding author on reasonable request. 


\section{Competing interests}

The author declares that he has no competing interests.

Received: 22 July 2020 Accepted: 5 February 2021

Published online: 17 February 2021

\section{References}

1. Fukui K, Nishikawa K, Ikeo S, Shintaku E, Takada K, Takanobu H, Honda M, Takanishi A (2005) Development of a talking robot with vocal cords and lips having human-like biological structures. In: Proceedings of the 2005 IEEE/RSJ international conference on intelligent robots and systems. pp 2526-2531. https://doi.org/10.1109/IROS.2005.1545356

2. Fukui K, Ishikawa Y, Ohno K, Sakakibara N, Honda M, Takanishi A (2009) Three dimensional tongue with liquid sealing mechanism for improving resonance on an anthropomorphic talking robot. In: Proceedings of the 2009 IEEE/RSJ international conference on intelligent robots and systems. pp 5456-5462 https://doi.org/10.1109/IROS.2009.5353983

3. Yoshikawa Y, Asada M, Hosoda K, Koga J (2003) A constructivist approach to infants' vowel acquisition through mother-infant interaction. Connect Sci 15(4):245-258. https://doi.org/10.1080/09540090310001655075

4. Takanobu H, Nakamura K, Takanishi A, Ohtsuki K, Ozawa D, Ohnishi M, Okino A (2001) Human skull robot as a mechanical patient simulator for mouth opening and closing training. In: Proceedings of the 2001 IEEE/RSJ international conference on intelligent robots and systems. pp 2154-2159. https://doi.org/10.1109/IROS.2001.976389

5. Jung J, Penning RS, Ferrier NJ, Zinn MR (2011) A modeling approach for continuum robotic manipulators: Effects of nonlinear internal device friction. In: Proceedings of the 2011 IEEE/RSJ international conference on intelligent robots and systems. pp 5139-5146. https://doi.org/10.1109/ IROS.2011.6094941

6. Hsiao K, Mochiyama H, Takei T, Shinotsuka E, Ogawa K (2017) Continuum manipulator with rubber skin layer including pulling-wire mechanism. J Robot Soc Jpn 35(3):221-229. https://doi.org/10.7210/jrsj.35.221 (in Japanese)

7. Kikuchi S, Yamada Y, Higashi R, Morita T (2015) Super multi-joint manipulator by using creased plate and pneumatic actuators arranged antagonistically. J Robot Mechatr 27(3):276-285. https://doi.org/10.20965 /jrm.2015.p0276

8. Sawada K, Osuka K, Ono T (1999) On soft machine-toward the realization of mechanical speech synthesizer -. In: Proceedings of the 1999 IEEE international conference on systems, man, and cybernetics. pp 978-983. https://doi.org/10.1109/ICSMC.1999.812543

9. Lu X, Xu W, Li X (2017) A soft robotic tongue-mechatronic design and surface reconstruction. IEEE/ASME Trans Mechatr 22(5):2102-2110. https ://doi.org/10.1109/TMECH.2017.2748606

10. Kataoka M, Yoshida T, Kojima T, Endo N, Asada M (2016) Development of a flexible tongue mechanism zets by using flexible pneumatic actuators. In:
Proceedings of the 17th SICE system integration division annual conference. pp 741-745. (in Japanese)

11. Hofe R, Moore RK (2008) Towards an investigation of speech energetics using 'anton': an animatronic model of a human tongue and vocal tract. Connect Sci 20(4):319-336. https://doi.org/10.1080/09540090802413251

12. Endo N, Kojima T, Endo K, lida F, Hashimoto K, Takanishi A (2013) Development of anthropomorphic soft robotic hand wsh-1 rii. In: Romansy 19-robot design, dynamics and control. pp 175-182. https://doi. org/10.1007/978-3-7091-1379-0_22

13. Lee J, Chung YS, Rodrigue H (2019) Long shape memory alloy tendonbased soft robotic actuators and implementation as a soft gripper. Sci Rep 9:11251. https://doi.org/10.1038/s41598-019-47794-1

14. Endo N (2020) Wire-pulling mechanism with embedded soft tubes for robot tongue. In: Proceedings of the 23rd CISM IFToMM symposium on robot design, dynamics and control. pp 313-320. https://doi. org/10.1007/978-3-030-58380-4_38

15. Webster RJ, Jones BA (2010) Design and kinematic modeling of constant curvature continuum robots: a review. Int J Robot Res 29(13):1661-1683. https://doi.org/10.1177/0278364910368147

16. Endo N, Kojima T, Ishihara H, Horii T, Asada M (2014) Design and preliminary evaluation of the vocal cords and articulator of an infant-like vocal robot "lingua". In: Proceedings of the 2014 IEEE-RAS international conference on humanoid robots. pp 1063-1068. https://doi.org/10.1109/ HUMANOIDS.2014.7041496

17. Endo N, Kizaki Y, Kamamichi N (2020) Flexible pneumatic bending actuator for a robotic tongue. J Robot Mechatr 32(5):894-902. https://doi. org/10.20965/jrm.2020.p0894

18. Mishra AK, Mondini A, Dottore ED, Sadeghi A, Tramacere F, Mazzolai B (2018) Modular continuum manipulator: analysis and characterization of its basic module. Biomimetics 3(1):3. https://doi.org/10.3390/biomimetic s3010003

\section{Publisher's Note}

Springer Nature remains neutral with regard to jurisdictional claims in published maps and institutional affiliations. 